\title{
Using algae components to mitigate intestinal damage after chemotherapy
}

Pengfei Zhang

Jing Liu

Bohui Xiong

Cong Zhang

Beining Kang

Yishan Gao

Zengkuan Li

Wei Ge

Shunfeng Cheng

Yanan Hao

Wei Shen

Shuai Yu

Liang Chen

Xiangfang Tang

Yong Zhao

Hongfu Zhang

\section{Video Byte}

Keywords: intestine, intestinal mucositis, chemotherapy, algae, AOS, Microbiome

Posted Date: November 11th, 2020

DOI: https://doi.org/10.21203/rs.3.rs-106074/v1

License: (c) (i) This work is licensed under a Creative Commons Attribution 4.0 International License.

Read Full License 


\section{Abstract}

Chemotherapeutic drugs can be a necessary part of cancer treatment, but side effects remain a major concern. Intestinal mucositis is an adverse effect of chemotherapy medicine that causes digestive distress. Although efforts have been made to minimize the effects of chemotherapy on the gut, more effective preventive measures are needed. A recent study examined the effects of fecal microbiota transplantation on intestinal function in patients receiving chemotherapy. Following up on their previous observation that an algae byproduct, alginate oligosaccharides (AOS), can increase beneficial gut microbes in mice, researchers treated healthy mice with AOS before transplanting their fecal microbes to mice treated with chemotherapeutics. They found that microbes from AOS-treated mice improved small intestine function better than microbes from control mice. Beneficial microbes were increased after transplantation, resulting in increased blood metabolites from digestion. Further studies with isolated microbes are needed to determine which microbial species are responsible for the effect of AOS, but the results suggest that treating donors with AOS before fecal microbiota transplantation may improve intestinal recovery in recipients. helping to effectively prevent intestinal mucositis after chemotherapy. 